\title{
ESTADO DE MAL EPILÉPTICO EM CRIANÇAS: EXPERIENCIA CLINICA COM O DIFENILIDANTOINATO DE SÓDIO POR VIA PARENTERAL
}

\author{
ANTRANIK MANISSADJian * \\ Hedda Arminante de O. Penna* \\ ANTONIO B. LEFÈvRE **
}

A observação no Pronto Socorro de Pediatria de casos fatais ocorridos em crianças em estado de mal - provàvelmente conseqüentes à depressão respiratória determinada pelo emprêgo endovenoso de medicamento anticonvulsivante tipo barbitúrico - despertou nossa atenção para a utilização de um medicamento anticonvulsivante, o difenilidantoinato de sódio, que preenche os seguintes requisitos: 1) controla as crises convulsivas sem determinar depressão respiratória; 2) não aprofunda o estado de inconsciência; 3) pode ser utilizado por via parenteral; 4) tem elevado índice de eficiência.

Murphy e Schwab ${ }^{1}$, em 1956, publicaram o primeiro trabalho clínico que relata a experiência de 5 anos do Massachussets General Hospital com o emprêgo parenteral dessa droga na profilaxia e no contrôle de crises convulsivas de pacientes submetidos a intervenções neurocirúrgicas e em pacientes em estado de mal. Os autores não observaram reações nos locais da aplicação venosa ou muscular e se preocuparam apenas em mostrar a boa tolerância do medicamento, não fornecendo dados estatísticos sôbre os seus resultados.

Concomitantemente, Dill e col. (citados por Schwab e Murphy ${ }^{3}$ ) verificaram, em pacientes que recebiam $300 \mathrm{mg}$ de difenilidantoinato de sódio por via oral, que o nível sangüíneo máximo era alcançado entre 2-8 horas, assim permanecendo durante 24 horas. Segundo Chen (citado por Schwab e Murphy ${ }^{3}$ ), em 30-40 minutos o nível sangüineo máximo é atingido pela administração de doses adequadas por via venosa.

Trabalho apresentado no Departamento de Neurologia da Associação Paulista de Medicina em 5 abril 1961: * Assistentes de Clinica Pediátrica (Prof. Pedro de Alcântara); ** Livre-Docente da Clinica Neurológica (Prof. A. Tolosa) da Fac. Med. da Univ. de São Paulo.

Nota dos autores - Este trabalho foi realizado com a colaboração da equipe de plantonistas do Pronto Socorro de Pediatria: Drs. Bettina de Camargo, Dácio Pinheiro, Fernando P. Gouvêa, Francisco F. De Fiore, Jácobo Bacal, José Araujo, Lucy Duailibi, Maria Aparecida Pereira, Mauricio Grinberg, Milton Zaidan, Paulo Eiró Gonçalves e Ruy Cesar F. Denunci. Agradecemos a colaboração do Dr. Roberto Pasqualin, do Laboratório Paulista de Biologia, pelo preparo e fornecimento do medicamento utilizado. 
Não há estudos sôbre a absorção pela via muscular, tendo-se, no entanto, a impressão de que deve ser a mesma que por via digestiva. Portanto, é evidente que a via de eleição para a utilização do medicamento para o tratamento do estado de mal é a via venosa.

Carter $^{2}$, em 1958, observou os efeitos da droga em 85 pacientes (4 com 5 anos ou menos, 6 com mais de 21 anos e o restante com idade variável entre 5 e 21 anos) com 121 estados de mal, utilizando $250 \mathrm{mg}$ por via venosa à razão de $50 \mathrm{mg}$ por minuto, com os seguintes resultados: em 66 pacientes, 87 crises convulsivas foram controladas por uma aplicação; em 14 pacientes, 17 crises convulsivas foram controladas por duas aplicações; em 5 pacientes, para o contrôle de 7 crises convulsivas, foi necessária a associação com barbitúrico. Carter aconselha o prosseguimento da administração do medicamento por via oral após a recuperação da consciência, não tendo notado qualquer efeito colateral. Os efeitos colaterais citados por Schwab e Murphy ${ }^{3}$ - ataxia e nistagmo ocorrendo 30-40 minutos após a injeção e persistindo por 24 horas - constituem a confirriação clínica do trabalho de Chen em ratos a respeito do nivel sangüineo. Lesões cerebelares discutìvelmente atribuídas ao emprêgo do difenilidantoinato de sódio foram descritas, apenas em um caso, por Hofmann (citado por Schwab e Murphy ${ }^{3}$ ).

A dose empregada em crianças seria, segundo Doge (citado por Schwab e Murphy ${ }^{3}$ ), de $200 \mathrm{mg} / \mathrm{m}^{2} /$ dia, o que daria $300 \mathrm{mg} /$ dia para adultos.

\section{METTODO, MATERIAL E RESULTADOS}

Utilizamos os seguintes esquemas de tratamento: $a$ ) para crianças até 1 ano de idade, $100 \mathrm{mg}$ por via venosa, injetados em tempo não inferior a $50 \mathrm{mg} / \mathrm{min}$.; $b$ ) para crianças maiores, $150-250 \mathrm{mg}$ à razâo de $50 \mathrm{mg} / \mathrm{min}$.

No periodo de outubro 1960 a janeiro 1961, o medicamento foi utilizado em 71 crianças em estado de mal, com idades compreendidas entre 3 dias e 10 anos. Destas, 6 faleceram em conseqüência da doença primária e não fazem parte da presente análise. Obtivemos $72,3 \%$ de resultados positivos (contrôle do estado de mal apenas com difenilidantoinato de sódio) e 27,7\% de resultados considerados negativos, quando houve necessidade de complementação com barbitúrico.

Correlacionando os resultados com os agrupamentos etários, obtivemos os dados que constam do quadro 1.

\begin{tabular}{|c|c|c|c|c|}
\hline \multirow{2}{*}{$I d a d e$} & \multicolumn{2}{|c|}{ Resultados positivos } & \multicolumn{2}{|c|}{ Resultados negativos } \\
\hline & $N^{o}$ & Percentagem & $N^{o}$ & Percentagem \\
\hline 0 a 3 semanas & 2 & 100,0 & _- & - \\
\hline 3 semanas a 12 meses. & 16 & 72,7 & 6 & 27,3 \\
\hline 1 a 3 anos $\ldots \ldots \ldots$ & 15 & 68,1 & 7 & 21,0 \\
\hline 3 a 6 anos $\ldots \ldots \ldots$ & 9 & 75,0 & 3 & 25,0 \\
\hline 6 a 10 anos $\ldots \ldots \ldots$ & 5 & 71,4 & 2 & 28,6 \\
\hline Totais & 47 & 72,3 & 18 & 27,7 \\
\hline
\end{tabular}


Não foi observada influência da presença ou ausência de hipertermia (quadro 2).

\begin{tabular}{|c|c|c|c|c|}
\hline & & \multicolumn{2}{|c|}{ Hipertermia } & \multirow{2}{*}{ Totais } \\
\hline & & Presente & Ausente & \\
\hline \multirow{2}{*}{ Resultad } & Positivos $\ldots \ldots \ldots \ldots \ldots$ & 22 & 25 & 47 \\
\hline & Negativos $\ldots \ldots \ldots \ldots \ldots$ & 9 & 9 & 18 \\
\hline \multicolumn{2}{|l|}{ Totais } & 31 & 34 & 65 \\
\hline
\end{tabular}

Quadro 2 - Correlação entre os resultados e a presença, ou não, de hipertermia.

Procuramos avaliar o efeito do medicamento correlacionando a duração da crise convulsiva com os resultados obtidos nos diferentes agrupamentos etários. Esta correlação é apresentada no gráfico 1 , onde se vê que a maior parte dos casos fol atendida nas primeiras 6 horas, a partir do inicio da crise.

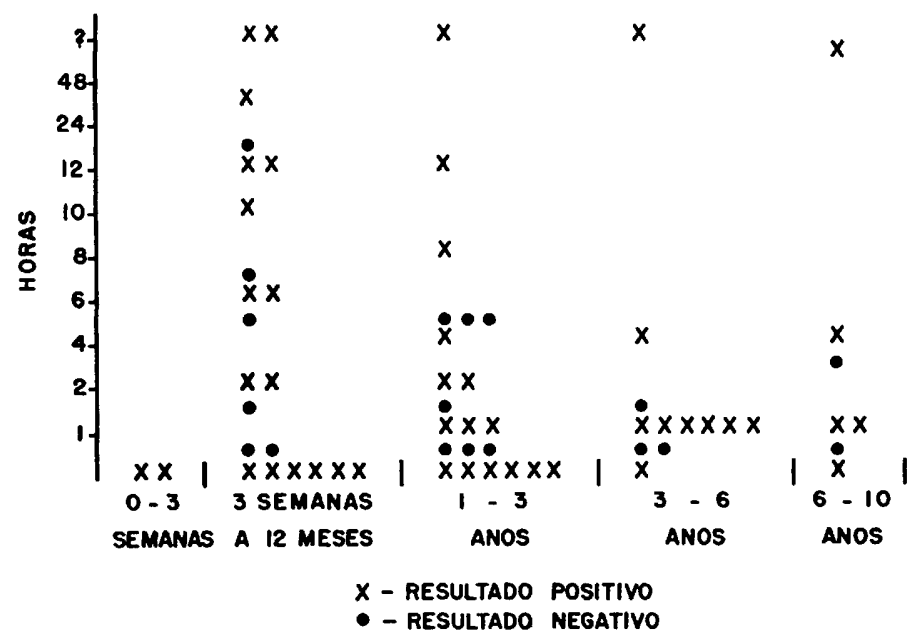

Gráfico 1 - Efeitos do difenilidantoinato de sódio avaliados pela correlação, nos diferentes agrupamentos etários, entre os resultados e o tempo decorrido a partir do inicio da crise.

Finalmente, procuramos estabelecer, nos casos em que os resultados foram positivos, a relação entre o tempo decorrido desde a administração do medicamento até a cessação das convulsōes nos diferentes agrupamentos etários (gráfico 2), notando-se que $85,1 \%$ dêsses resultados foram observados nos primeiros 20 minutos; em $50 \%$ dêstes últimos casos a cessação da crise ocorreu durante a administração do medicamento. 


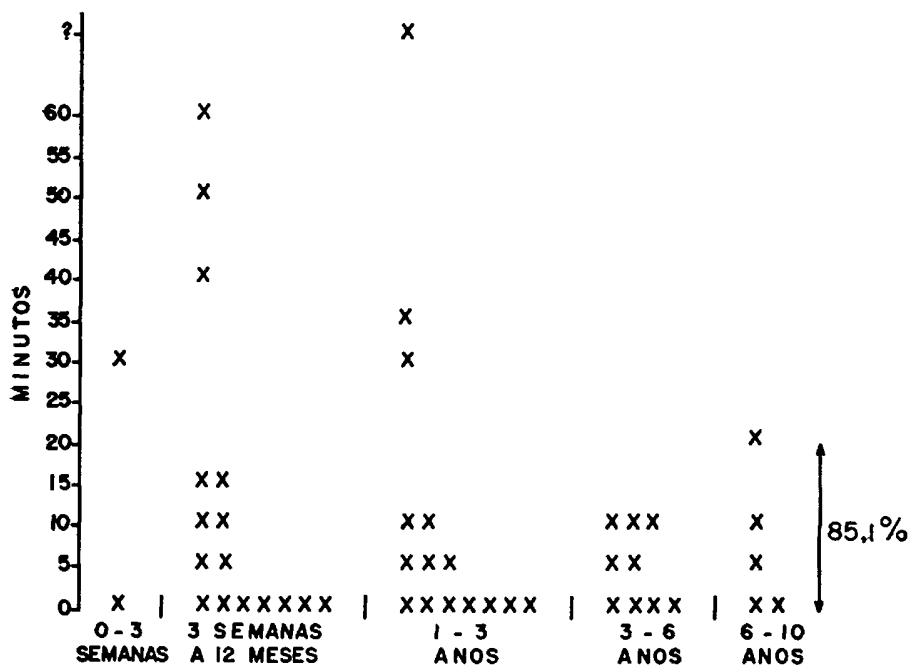

Gráfico 2 - Relação entre o tempo decorrido entre o início da administração do difenilidantoinato de sódio e a cessacão das convulsões nos diferentes agrupamentos etários.

\section{CONCLUSÕES}

1. O difenilidantoinato de sódio é inócuo, não tendo sido observados efeitos colaterais que possam contra-indicar o seu uso; o nistagmo transitório que pode ser observado durante o seu emprêgo deve ser interpretado como indicativo de ação medicamentosa sôbre o sistema nervoso central.

2. A idade das crianças e a presença ou ausência de hipertermia não influíram nos resultados observados.

3. Na grande maioria dos casos que se beneficiaram a resposta terapêutica ocorreu nos primeiros 20 minutos a partir do início da administração; deve ser salientado que na metade dêstes casos a crise cessou durante a administração do medicamento.

\section{RESUMO}

Os autores realizaram experiência clínica empregando o difenilidantoinato de sódio em crianças com estado de mal epiléptico. Analisam os resultados da ação do medicamento em função da idade, tempo de duração do estado de mal e presença, ou não, de hipertermia. Concluem pela inocuidade do medicamento e pela sua eficiência em um número de casos $(72,3 \%)$ suficiente para justificar a sua utilização como recurso terapêutico de escolha em casos de estado de mal epiléptico. 


\section{SUMMARY \\ Status epilepticus in children: experience with parenteral sodium diphenylhydantoin.}

Report on the use of sodium diphenylhydantoin in the management of status epilepticus in children. The data obtained are correlated with age groups, duration of the status epilepticus and hyperthermia. The results indicate that the drug is safe and efficient in a large number of cases $(72.3 \%)$, justifying its use as a choice drug.

\section{REFERENCIAS}

1. MURPHY, J. T.; SCHWAB, R. S. - Diphenylhydantoin (Dilantin) sodium used parenterally on control convulsions: a five year report. J.A.M.A., 160:385-388 (fevereiro, 4) 1956. 2. CARTER, C. H. - Use of parenteral Diphenylhydantoin (Dilantin) sodium in control of status epilepticus. AMA Arch. Neurol. a. Psychiat, 79:136-137, 1958. 3. SCHWAB, R. S.; MURPHY, J. T. - Recent experiences with parenteral Dilantin. Epilepsia, 1:227-231, 1960.

Clínica Pediátrica - Hospital das Clinicas da F'ac. Med. da Univ. de São Paulo - Caixa Postal 8091 - São Paulo, Brasil. 